\title{
Clear anti-correlation between luminosity and high energy cutoff in the low/hard state of the black hole candidate GX339-4
}

\author{
Takehiro G. Miyakawa*, Kazutaka Yamaoka, Atsumasa Yoshida, Koji Saito, \\ Department of Physics and Mathematics, Aoyama Gakuin University, Fuchinobe 5-10-1, \\ Kanagawa 229-8558, Japan \\ E-mail: tmiyakawa@phys.aoyama.ac.jp, yamaoka@phys.aoyama.ac.jp
}

\section{Tadayasu Dotani, and Hajime Inoue}

The Institute of Space and Astronautical Science, Japan Aerospace Exporlation Agency,

Yoshinodai 3-1-1, Sagamihara, Kanagawa 229-8510, Japan

\begin{abstract}
We have analyzed the 171 RXTE data sets of the black hole candidate GX 339-4 in the low/hard state during its 1996-2005 outburst. All the broadband spectra were successfully modeled by a simple analytic model, power-law with an exponential cutoff. The obtained energy cutoff $\left(E_{\text {cut }}\right)$ is distributed over $50-300 \mathrm{keV}$, and the photon index over 1.4-1.6. We found a clear correlation ( $E_{\text {cut }}$ is proportional to $L^{-0.75 \pm 0.04}$ ) between luminosity in $2-200 \mathrm{keV}(\mathrm{L})$ and $E_{\text {cut }}$ when $\mathrm{L}$ is larger than $5 \times 10^{37}$ erg $s^{-1}$, while $E_{\text {cut }}$ is almost constant at $200 \mathrm{keV}$ when L is smaller than $5 \times 10^{37}$ erg $s^{-1}$. This anti-correlation is unchanged by adopting the more physical model of thermal Comptonization by Sunyaev and Titarchuk, although a slightly different relation is obtained as the electron temperature is proportional to $L^{-0.23 \pm 0.02}$. These anti-correlations are qualitatively explained by a picture where the energy flow rate from protons to electrons balance with cooling due to inverse Compton scattering.
\end{abstract}

VI Microquasar Workshop: Microquasars and Beyond September 18-22, 2006

Como, Italy

\footnotetext{
${ }^{*}$ Speaker.
} 


\section{Introduction}

Energy spectra of the black hole candidates (BHCs) have mainly two spectral states: high/soft state and low/hard state. It has been established that the high/soft state happens at comparatively high mass accretion rate, and there is an optically thick and geometrically thin accretion disk which extends to three Schwarzschild radii from the central black hole. However, the origin of the low/hard state has not been clarified yet. X-ray spectra in the low/hard states are well represented by a power law with a photon index of 1.4 1.7 (Tanaka \& Shibazaki 1996), The energy cutoff of the spectrum $\left(E_{\text {cut }}\right)$ is seen at $\sim 100 \mathrm{keV}$ by past gamma-ray observations (Grove et al. 1998). It is believed that there is a high temperature corona inside the standard disk, and radiation is produced by inverse Compton scattering from a part of soft photons via optically thin plasma with very high temperature around the black hole. Indeed, the broadband spectra of many black hole binaries in the hard state are successfully modeled by thermal Comptonization model (Dove et al. 1996, Pountanen \& Svensson 1996), although location and geometry of the corona are still debated.

GX 339-4 was found by the X-ray satellite OSO-7 in 1971 (Markert et al. 1973). Since it was similar to Cygnus $\mathrm{X}-1$ in terms of the feature of spectrum and short time variations, it was suggested as a black hole candidate (Samimi et al. 1979). This source is one of the best studied BHCs at X rays and gamma rays by various instruments: Ginga/LAC (Ueda, Ebisawa and Done 1994), CGRO/OSSE(Grabelsky et al. 1995, Smith et al. 1996), ASCA (Wilms et al. 1998), RXTE (Smith et al. 1996), and Beppo-SAX (Corongiu et al. 2003). GX 339-4 exihited the five different spectral states in the previous outbursts (Tanaka \& Shibazaki 1996), but often stayed in the low/hard state. All the spectra in the low/hard state are roughly explained by a power law with an exponential cutoff or thermal Comptonization model, requiring some modification of reflection component, soft excess and iron-K lines. Detailed broadband analysis in 2-1000 keV was done using the Ginga, RXTE and OSSE data in 1991 and 1996 observations by Zdziarski et al. (1999) and Wardziński et al. (2002). Wardziński et al. (2002) concluded that the four spectra in the hard state have very similar intrinsic $X$-ray slope of photon index $\cong 1.75$. On the other hand, they found a possible correlation that the high-energy cutoff energy decreases with luminosity increasing. Zdziarski et al. (2004) studied the long term behaviour using 16-years light curves by compiling the GINGA/ASM, CGRO/BATSE, and RXTE/ASM data, and also found that the electron temperature depends on the luminosity by showing a correlation between the BATSE flux and photon index in the $20-160 \mathrm{keV}$ range.

As mentioned above, many authors have studied some observations during individual outbursts of this source. Hence, the correlation among various parameters (high energy cutoff, photon index, and so on) are poorly understood. Thus, in order to clarify the radiation mechanism of the low/hard state, we first performed a systematic study of detailed correlations among spectral parameters using the large public RXTE data archives.

In this paper, we report on the results of the analysis of 171 RXTE data sets of GX 339-4 in the low/hard state during its 1996-2005 outburst. In Section 2, we describe the procedure of analysis. In section 3, we present the results and discussions. 

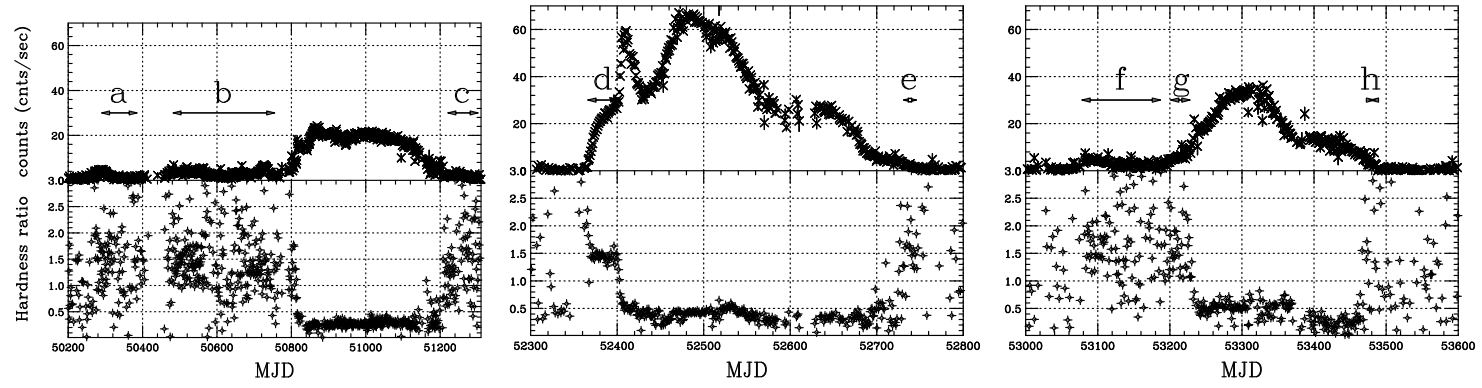

Figure 1: RXTE/ASM light curve and hardness ratio of GX 339-4 from 1996 to 2005.
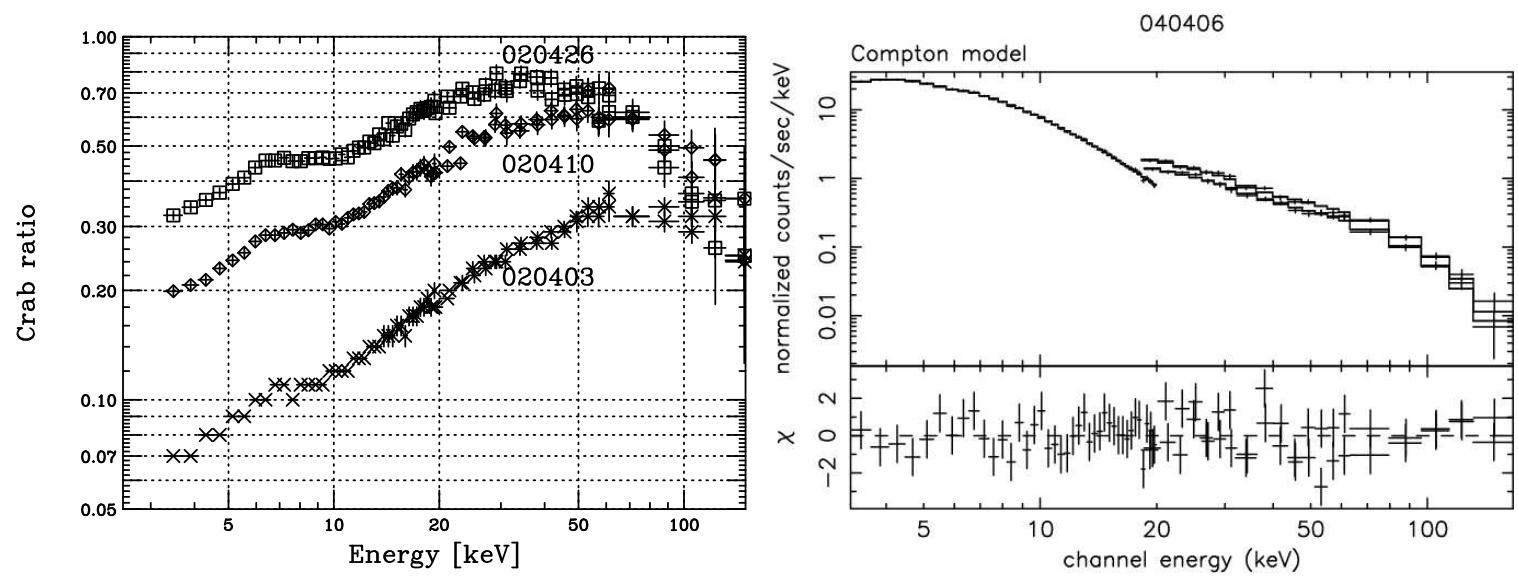

Figure 2: Left: PHA ratio of GX 339-4 to Crab taken during different epochs. Right: The results of spectrum analysis observed in 6th April 2004. (Low energy side is PCA. The high energy side is clusterA and clusterB. This analysis was done with the Compton model.)

\section{Observations and Data Reduction}

GX 339-4 has been observed over $\sim 10$ years by RXTE satellite. Figure 1 shows the 1.5$12 \mathrm{keV}$ RXTE/ASM light curve. GX 339-4 underwent three outbursts in 1997, 2002, and 2004 during the past 10 years. According to McClintock \& Remillard (2004), it is known that HR2 in the low/hard state shows $\sim 1.5$ where HR2 is the ratio of ASM counts in $5-12 \mathrm{keV}$ to counts in $3-5 \mathrm{keV}$. Base on this criterion, we selected the data during the hard state from the publicly available RXTE data archive at NASA/HEASARC. Thus, we obtained 171 RXTE data sets indicated by arrows in Figure1. For the RXTE data reduction, we used the publicly available software HEADAS 6.0.2 provided by NASA/GSFC.

We used standard 2 mode data which has a time resolution of $16 \mathrm{sec}$ and 129 energy channels covering the full range of the PCA detectors. The PCA background was estimated with the background model for bright sources. As for the HEXTE, we used the archive mode data with a time resolution of $16 \mathrm{sec}$ taken from both cluster A and B, and subtracted the background taken from the rocking motion. Two spectral models are applied to all the data in carrying out the systematic spectral analysis of the continuum. One is a simple analytic model, power-law with an exponential cutoff (cutoff power-law), and the other is thermal Comptonization model introduced by Sunyaev \& Titarchuk (1980) (compst in XSPEC). The cutoff power-law model consists of two 

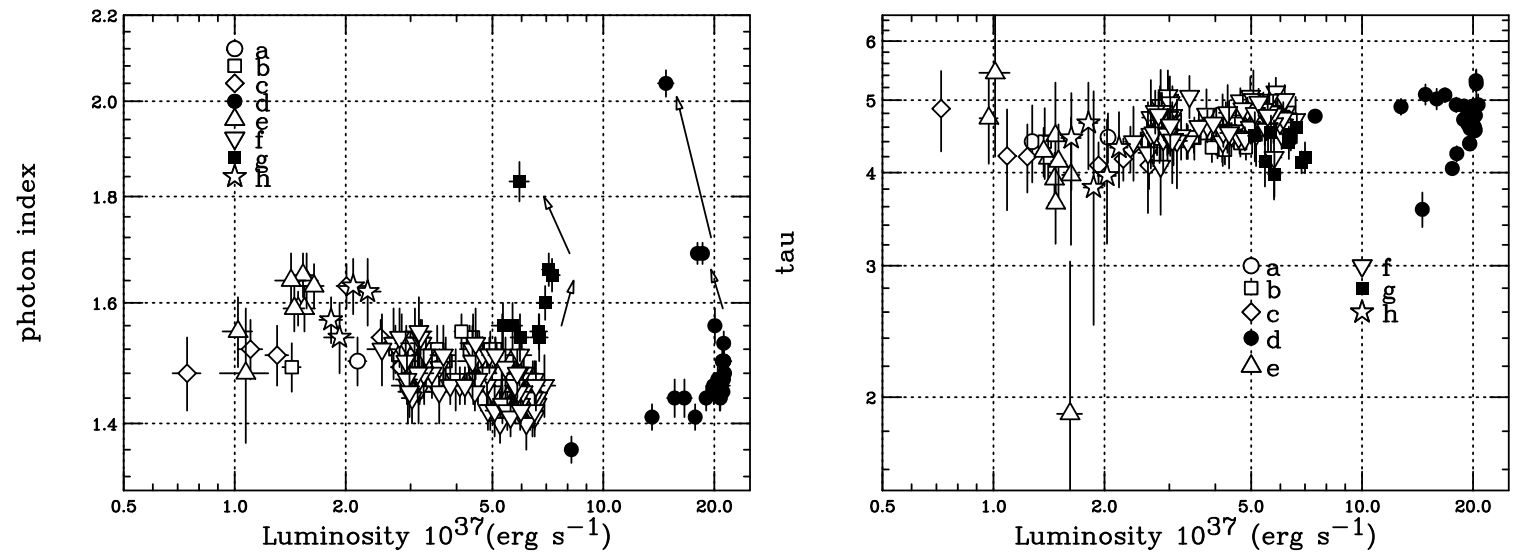

Figure 3: Left: relation between luminosity in $2-200 \mathrm{keV}$ and photon index. Right: relation between luminosity and optical depth

parameters: a photon index $(\alpha)$ and an energy of spectrum cutoff $\left(E_{\text {cut }}\right)$. The compst model also has two parameters: an electron temperature $\left(\mathrm{k} T_{\mathrm{e}}\right)$ and a Thomson optical depth $(\tau)$ of high temperature plasma. These continuum models are further modified by galactic absorption (wabs in XSPEC) and smeared edge model (Ebisawa et al. 1994). The hydrogen column density was fixed to $5 \times 10^{21} \mathrm{~cm}^{-2}$ (Ilovaisky et al. 1986). The energy of the absorption edge and width in the smeared edge model is fixed at $7.11 \mathrm{keV}$ due to the neutral iron-K edge and $10 \mathrm{keV}$, respectively. We added $2 \%$ systematic errors to each PCA spectral bin so as to $\chi_{v}{ }^{2} \sim 1$ for the Crab nebula. The PCA energy range is limited to $3 \sim 20 \mathrm{keV}$ bacause HEXTE's effective area is larger than that of PCA above $20 \mathrm{keV}$. We fit the data of PCA and HEXTE in 3-200 keV. simultaneously, leaving the relative normalization of PCA and HEXTE to be free. The HEXTE normalization factor is found to be always $0.90-0.95$. The current PCA and HEXTE response matrices give a consistent value of photon index with $2.08 \pm 0.02$ in $3-20 \mathrm{keV}$ and $2.10 \pm 0.02$ in $20-200 \mathrm{keV}$ for the Crab Nebula, which is applicable to the joint fitting between the two instruments. An X-ray luminosity (L) was calculated based on the PCA flux over 2-200 keV assuming the distance to GX 339-4 at $8 \mathrm{kpc}$ (Zdziarski et al 2004). The obtained luminosity ranges from $7 \times 10^{36} \mathrm{erg} \mathrm{s}^{-1}$ to $2.1 \times 10^{38} \mathrm{erg} \mathrm{s}^{-1}$, corresponding to $2-20 \%$ of the Eddington limit $\left(L_{\mathrm{E}}\right)$ assuming the black hole mass at $10 M_{\odot}$.

\section{Results and discussions}

In order to ascertain the presence of a high energy cutoff in a model-independent way, we first calculated the PHA ratio of GX 339-4 to the Crab Nebula. The Crab spectrum was extracted in the same way as above from data observed on April 28th 2002. A Crab spectrum in 3-200 keV was represented by a simple featureless power-law model with a photon index of $2.10 \pm 0.01$. The left side of Figure 2 shows the spectrum ratio of GX 339-4 to Crab. The spectra are from April 3th 2002 (MJD 52367), April 10th 2002 (MJD 52374), and April 26th 2002 (MJD 52390), respectively. As can be seen from this figure, we found a clear high energy cutoff at $\sim 30 \mathrm{keV}, \sim 50 \mathrm{keV}$, and $\sim 70$ $\mathrm{keV}$ from upper to lower, suggesting that the peak energy varies with the X-ray luminosity, while the spectral slope, i.e. photon index, does not seem to vary significantly.

The right panel of Figure 2 is sample in analyzing the spectrum taken on April 6 2004. Both models 

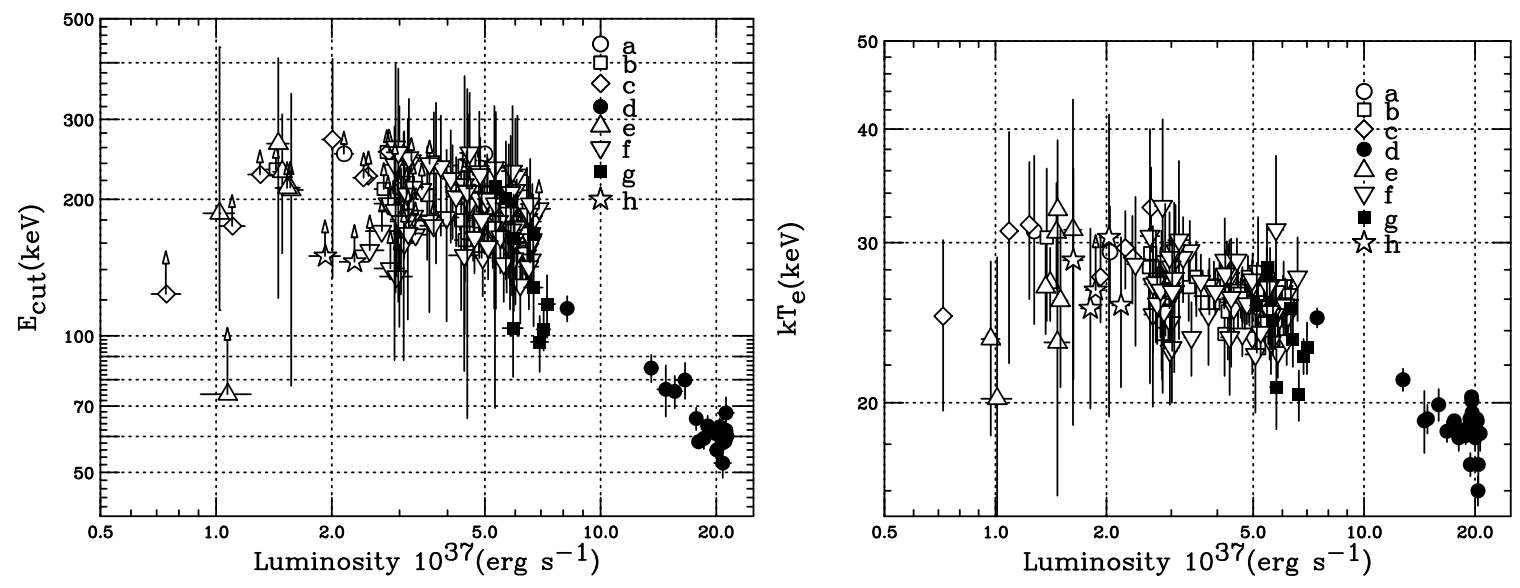

Figure 4: Left: Relation between luminosity in $2-200 \mathrm{keV}$ and energy cutoff $\left(E_{\text {cut }}\right)$. The results are for a fit with a cutoff power law model. Right: Relation between the electron temperature $\left(k T_{\mathrm{e}}\right)$ and luminosity in 2-200 keV. The results are from the COMPST model.

can represent the broadband spectrum over 3-200 keV successfully.

The left panels of Figure 3 and Figure 4 show the relation between parameters of the cutoff power law model. The points correspond from the different outbursts. The high energy cutoff $\left(E_{\text {cut }}\right)$ ranges from $50 \mathrm{keV}$ to $300 \mathrm{keV}$ or more where we cannot constrain the parameters well. As you can see from this figure, we found a clear anti-correlation between $L$ and $E_{\text {cut }}$ when $\mathrm{L}>5 \times 10^{37} \mathrm{erg} \mathrm{s}^{-1}$. This relation follows an equation: $E_{\text {cut }} \propto L^{-0.75 \pm 0.04}$. Errors quoted are at the $90 \%$ confidence level. On the other hand, $E_{\text {cut }}$ seems to be almost constant at $200 \mathrm{keV}$ when $\mathrm{L}<5 \times 10^{37} \mathrm{erg} \mathrm{s}^{-1}$. Furthermore, we found that the epoch when the X-ray luminosity is larger than $7 \times 10^{37} \mathrm{erg} \mathrm{s}^{-1}$ corresponds to an initial rising phase of the outburst in 2002 and 2004. Unlike the high energy cutoff, the photon index is not sensitive to the X-ray luminosity. It is distributed over 1.4-1.7, which is consistent with the typical value of black hole candidates in the low/hard state. When the source becomes brighter than a certain value in the initial phase, the photon index shows 1.8-2.1. This may be considered as a part of state transitions from hard state to intermediate or very high state.

In the right panel of Figure 3 and Figure 4, we further show correlations between the parameters when we fit the spectra with a more physical component: the thermal Compton model of Sunyaev \& Titarchck. $\mathrm{k} T_{\mathrm{e}}$ is distributed over $20-30 \mathrm{keV}$, while the optical depth $\tau$ is distributed over 4 5. We also found a clear anti-correlation between of $\mathrm{k} T_{\mathrm{e}}$ and $L$ of $k T_{\mathrm{e}} \propto L^{-0.23 \pm 0.02}$ when $\mathrm{L}>5 \times 10^{37}$ $\mathrm{erg} \mathrm{s}^{-1}$. The $\mathrm{k} T_{\mathrm{e}}$ remains at $26 \mathrm{keV}$ when $\mathrm{L}<5 \times 10^{37} \mathrm{erg} \mathrm{s}^{-1}$.

Next, We will now try to explain the anti-correlation between $L$ and $\mathrm{k} T_{\mathrm{e}}$ quantitatively. Let us assume a high temperature corona with a spherical geometry (radius $\mathrm{R}$ ). The proton temperature $\left(\mathrm{k} T_{\mathrm{p}}\right)$ is assumed to be approximately constant at $\sim \frac{G M m_{\mathrm{p}}}{R}\left(m_{\mathrm{p}}\right.$ is the proton mass and $\mathrm{R}$ is the radius), that is, the energy loss rate of protons is much smaller than viscous heating rate through the accretion. The protons will give their energy to electrons through two-body collisions and the energy loss rate per unit volume is given as $\frac{\frac{3}{2} n k T_{\mathrm{p}}}{t_{\mathrm{pe}}}$ if $T_{\mathrm{p}}$ is much larger than $T_{\mathrm{e}}$ where $\mathrm{n}$ is 
a number density of plasma. $t_{\mathrm{pe}}\left(\propto \frac{\left(\frac{k T_{\mathrm{p}}}{m_{\mathrm{p}}}+\frac{k T_{\mathrm{e}}}{m_{\mathrm{e}}}\right)^{\frac{3}{2}}}{n}\right)$ is the equipartition time-scale due to Coloumb collision (Spizter 1962). The cooling rate per unit volume is approximately given as $\frac{4 k T_{\mathrm{e}}}{m_{\mathrm{e}} c^{2}} U_{\text {rad }} \mathrm{n} \sigma_{\mathrm{T}}$ where $c$ is the light speed, $U_{\text {rad }}$ is the photon flux density which is given by $U_{\text {rad }} \cong \frac{L \tau}{\pi R^{2}}$, and $\sigma_{\mathrm{T}}$ is the cross section of Thomson scattering. If $\frac{k T_{\mathrm{e}}}{m_{e}}$ is larger than $\frac{k T_{p}}{m_{p}}$, we will get $t_{p e} \propto \frac{T_{\mathrm{e}}^{\frac{3}{2}}}{n}$. In the steady state, the energy flow rate from proton to electron should balance with the cooling rate due to inverse Compton scattering, i.e. $\frac{\frac{3}{2} n k T_{\mathrm{p}}}{t_{\mathrm{pe}}}=\frac{4 k T_{\mathrm{e}}}{m_{\mathrm{e}} \mathrm{C}^{2}} U_{\mathrm{rad}} n \sigma_{\mathrm{T}}$. Using these equations, we can work out the anti-correlation of $k T_{\mathrm{e}} \propto L^{-\frac{2}{5}}$. Thus, the obtained anti-correlation is quantitatively explained by the fact that the radiation mechanism is due to inverse Compton scattering. Zdziarski et al. (1998) also suggest that the high energy cutoff depends on the luminosity from a theoretical point of view of thermal Comptonization. They showed a relation of $k T_{\mathrm{e}} \propto L^{-2 / 7}$ and $k T_{\mathrm{e}} \propto L^{-1 / 6}$ in the advection dominant and cooling dominant case, respectively. In comparison with this prediction, our result, $\sim-0.23$, is well consistent with these values. Furthermore, the maximum luminosity in the low/hard state is predicted as $L_{\max } \sim 0.15 y^{\frac{3}{5}} \alpha^{\frac{7}{5}} L_{\mathrm{E}}$ in his model. The highest luminosity observed in $2002, \sim 0.15 L_{\mathrm{E}}$, is quite agreement with this value.

\section{References}

[1] Corongiu, A., Chiappetti, L., Haardt, F., Treves, A., Colpi, M., and Belloni, T., 2003, A\&A, 408, 347

[2] Ebisawa, K.,Ogawa, M.,Aoki, T.,Dotani, T.,Takizawa, M.,Tanaka, Y., Yoshida, K.,Miyamoto, S.,Iga, S.,Hayashida, K., 1994, PASJ, 46, L375

[3] Grove J. E., Johnson W. N., Kroeger R. A., McNaron-Brown K., Skibo, J. G., Philips, B. F., 1998, ApJ, 500,L899

[4] Inoue, H., 1993 , in Multi-Wavelength Continuum Emission of AGN, Symposium 159, p73

[5] McClintock, J, E., Remillard, R, A., 2004, In Compact Stellar X-ray Sources. Cambridge, UK: Cambridge Univ. Press, 157

[6] Smith, I.A., Liang, E.P., Lin, D., Moss, M., Crider, A., Fender, R.P., Durouchoux, Ph., Corbel, S., and Sood, R., 1999, ApJ, 519, 762

[7] Spizter, L.Jr., 1962, Physics of Fully Ionized Gases(Wiley, New York)

[8] Sunyaev, R., A., Titarchuk, L., G., 1980, A\&A, 86, 121

[9] Tanaka, Y., Shibazaki, N., 1996 ARA\&A, 34, 607

[10] Yamaoka, K., Uzawa, M., Arai, M., Yamazaki, T., Atsumasa., Y, 2004 , Chinese Journal of Astronomy and Astrophysics , 2004, 5, L273

[11] Zdziarski, A.,Gierliński, M.,Mikolajewska, J.,Wardziński, G.,Smith,M, D., Harmon,A, B.,Kitamoto, S.,2004,ApJ,791 